Virginia Commonwealth University vCU Scholars Compass

\title{
Theoretical calculations of magnetic order and anisotropy energies in molecular magnets
}

M.R. Pederson

Naval Research Laboratory

D. V. Porezag

Naval Research Laboratory

J. Kortus

Naval Research Laboratory

S. N. Khanna

Virginia Commonwealth University

Follow this and additional works at: http://scholarscompass.vcu.edu/phys_pubs

Part of the Physics Commons

Pederson, M. R., Porezag, D. V., Kortus, J., et al. Theoretical calculations of magnetic order and anisotropy energies in molecular magnets. Journal of Applied Physics 87, 5487 (2000). Copyright (C) 2000 AIP Publishing LLC.

\section{Downloaded from}

http://scholarscompass.vcu.edu/phys_pubs/129

This Article is brought to you for free and open access by the Dept. of Physics at VCU Scholars Compass. It has been accepted for inclusion in Physics Publications by an authorized administrator of VCU Scholars Compass. For more information, please contact libcompass@vcu.edu. 


\title{
Theoretical calculations of magnetic order and anisotropy energies in molecular magnets
}

\author{
M. R. Pederson, D. V. Porezag, and J. Kortus \\ Center for Computational Materials Science - 6392, Naval Research Laboratory, \\ Washington, D.C. 20375-5000 \\ S. N. Khanna \\ Department of Physics, Virginia Commonwealth University, Richmond, Virginia 23284-2000
}

\begin{abstract}
We present theoretical electronic structure calculations on the nature of electronic states and the magnetic coupling in the $\mathrm{Mn}_{12} \mathrm{O}_{12}$ free cluster and the $\mathrm{Mn}_{12} \mathrm{O}_{12}(\mathrm{RCOO})_{16}\left(\mathrm{H}_{2} \mathrm{O}\right)_{4}$ molecular magnetic crystal. The calculations have been performed with the all-electron full-potential NRLMOL code. We find that the free $\mathrm{Mn}_{12} \mathrm{O}_{12}$ cluster relaxes to an antiferromagnetic cluster with no net moment. However, when coordinated by sixteen $\mathrm{HCOO}$ ligands and four $\mathrm{H}_{2} \mathrm{O}$ groups, as it is in the molecular crystal, we find that the ferrimagnetic ordering and geometrical and magnetic structure observed in the experiments is restored. Local Mn moments for the free and ligandated molecular magnets are presented and compared to experiment. We identify the occupied and unoccupied electronic states that are most responsible for the formation of the large anisotropy barrier and use a recently developed full-space and full-potential method for calculating the spinorbit coupling interaction and anisotropy energies. Our calculated second-order anisotropy energy is in excellent agreement with experiment. (C) 2000 American Institute of Physics.
\end{abstract}

[S0021-8979(00)65708-0]

Recently the $\mathrm{Mn}$-acetate crystal $^{1}$ has attracted a significant degree of experimental attention. ${ }^{2-7}$ However, there have only been a few computational studies on this molecule to date. ${ }^{8-12}$ The crystal consists of $\mathrm{Mn}_{12} \mathrm{O}_{12}(\mathrm{RCOO})_{16}\left(\mathrm{H}_{2} \mathrm{O}\right)_{4}$ molecules with $R=\mathrm{CH}_{3}$ and six additional molecules of solvation (four water and two acetic acid molecules). The unit cell has a volume of $3716 \AA^{3}$ and its ferrimagnetic core consists of a $\mathrm{Mn}_{12} \mathrm{O}_{12}$ cluster which has a total spin $S$ of 10 (moment of $20.0 \mu_{B}$ ). The magnetic $\mathrm{Mn}_{12} \mathrm{O}_{12}$ clusters in different cells are separated from one another by the nonmagnetic RCOO ligands, coordinated water molecules and the noninteracting molecules of solvation. The large separation prevents any significant exchange coupling between neighboring cores and the dipolar interaction between a molecule and its neighbors is less than $1 \mathrm{~K}$. As such the crystal is composed of a collection of noninteracting magnetic molecules which each have a large anisotropy energy. When placed in an applied magnetic field the hysteresis loops show staircase structure proposed to be associated with resonant quantum tunneling of spins between the different $M_{S}$ states. From an Arrhenius law, the magnetomolecular anisotropy barrier has been estimated to be around 60-62 K. ${ }^{5}$ However recent high-field ESR experiments by Barra et al. provide the required data to determine that the lowest (second-order) contributions to the barrier are $56.6 \mathrm{~K}$ which means that the higher-order contributions increase the barrier by less than $10 \% .^{6}$

In this work we report several recent theoretical calculations on the structure, chemistry, magnetic ordering, and anisotropy barriers for the $\mathrm{Mn}$-acetate molecule. We show that in addition to maintaining a large separation between the neighboring molecules, the ligands and coordinated water molecules are necessary for stabilizing the geometry of the magnetic core and that their presence significantly changes the magnitudes of the local moments on the Mn atoms. Calculations on the ligandated structure confirm the local magnetic moments suggested by experiments. To address the formation of the large anisotropy barrier we calculate the second-order anisotropy barrier using a new method for the inclusion of the spin-orbit interaction. ${ }^{10} \mathrm{We}$ also present the first calculations on the part of the higher-order contributions to the anisotropy energy that is entirely due to the spin-orbit coupling terms. These results suggest that the higher-order contributions to the anisotropy barrier are either due to electronic relaxations that are dependent on spin-orientation, higher-order couplings between scalar relativistic and spinorbit operators or possibly couplings between electronic and nuclear spins. The theoretical studies were carried out using a linear combination of atomic orbital expansion of the molecular orbitals within the density functional formalism. The atomic orbitals are expressed in terms of Gaussian basis functions and the exchange correlation effects are incorporated within the generalized gradient approximation ${ }^{13}$ (GGA) to the density functional theory. The calculations reported here were carried out using the Naval Research Laboratory Molecular Orbital Library (NRLMOL). Discussion of this methodology has appeared elsewhere ${ }^{14}$ and discussion specific to the $\mathrm{Mn}_{12} \mathrm{O}_{12}$ core (e.g., basis sets) appeared in Ref. 8 .

Pictured in Fig. 1 is the optimized structure for the $\mathrm{Mn}_{12} \mathrm{O}_{12}(\mathrm{RCOO})_{16}\left(\mathrm{H}_{2} \mathrm{O}\right)_{4}$ molecule. In Table I we compare some calculated $\mathrm{Mn}-\mathrm{Mn}$ separations to the experimentally determined separations. All bond lengths of our optimized ligandated structure are in excellent agreement with the experimentally determined geometry. In accord with experiment we find that the molecule has a net spin of $20 \mu_{B}$ and is ferrimagnetically ordered. The inner $\mathrm{Mn}_{1}$ atoms are minor- 


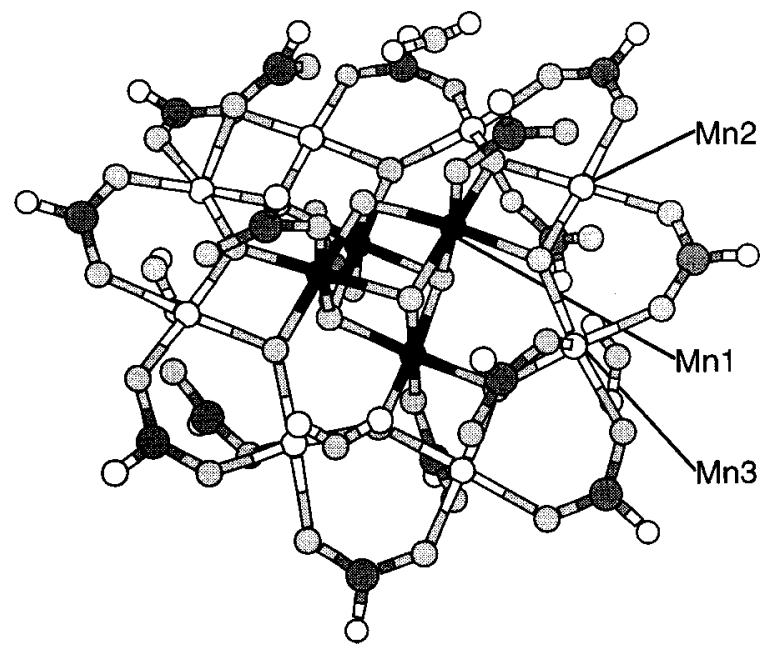

FIG. 1. Geometry of $\mathrm{Mn}_{12} \mathrm{O}_{12}(\mathrm{HOOC})_{16}\left(\mathrm{H}_{2} \mathrm{O}\right)_{4}$ determined from NRLMOL. The four black minority spin $\mathrm{Mn}_{1}$ atoms form a cube at the center of the molecule and the eight white majority spin $\left(\mathrm{Mn}_{2}\right.$ and $\left.\mathrm{Mn}_{3}\right)$ form a puckered $\mathrm{Mn}-\mathrm{O}$ ring around the inner cube. The $\mathrm{O}$ atoms (light gray) associated with the HOOC ligands and water molecules coordinate themselves with the Mn atoms so that each of the metal atoms is sixfold coordinated. The carbon atoms appear as dark gray and the hydrogen atoms appear as small off-white spheres.

ity spin atoms while the outer $\mathrm{Mn}_{2}$ and $\mathrm{Mn}_{3}$ atoms are majority spin electrons. To address the magnitudes of the local moments we have calculated the total moment enclosed within a sphere of radius $1.48 \AA$ and find that within these spheres, the inner minority spin atoms have a net spin of $-2.57 \mu_{B}$ while the outer majority spin atoms have a net spin of 3.58 and $3.63 \mu_{B}$, respectively. In other calculations we have ascertained that a $\mathrm{Mn}$ atom with five unpaired $d$-electrons will have a spin of approximately $4.1 \mu_{B}$ in a sphere of this size. Thus the experimental interpretations that the total local spins on the inner and outer Mn atoms are -3.0 and $4.0 \mu_{B}$, respectively, is reasonable. We have also removed the $16 \mathrm{HOOC}$ ligands and the $4 \mathrm{H}_{2} \mathrm{O}$ molecules and allowed the structure to relax further within the symmetry of the experimental structure. While the resulting structure retains the ferrimagnetic ordering the moments on the inner and outer atoms are found to be -4.1 and $4.2 \mu_{B}$, respectively. Further as shown in the table the geometry of the isolated $\mathrm{M}_{12} \mathrm{O}_{12}$ structure is significantly perturbed as compared to the ligandated structure. If a full relaxation, without symmetry constraints, is carried out we find that the isolated

TABLE I. Selected Mn-Mn separations for the ligandated and isolated $\mathrm{Mn}_{12} \mathrm{O}_{12}$ core. The ligands prevent bonding between the inner and outer $\mathrm{Mn}$ atoms. The passivated structure and the experimental passivated structure. All distances are in angstroms. The labels on each atom correspond to those shown in Fig. 1.

\begin{tabular}{lccc}
\hline \hline & & \multicolumn{2}{c}{ Ligandated } \\
\cline { 3 - 4 } Separation & Isolated theory & Theory & Experiment \\
\hline $\mathrm{Mn}_{1}-\mathrm{Mn}_{1}(\mathrm{~A})$ & 3.14 & 2.90 & 2.94 \\
$\mathrm{Mn}_{1}-\mathrm{Mn}_{1}(B)$ & 2.82 & 2.85 & 2.82 \\
$\mathrm{Mn}_{1}-\mathrm{Mn}_{3}$ & 2.60 & 3.48 & 3.45 \\
$\mathrm{Mn}_{1}-\mathrm{Mn}_{2}$ & 2.65 & 2.28 & 2.77 \\
\hline \hline
\end{tabular}

structure will prefer the four-layer $(\mathrm{MnO})_{3}$ hexagonal tower structure that has been observed in the gas phase by Castleman and Ziemann. ${ }^{15}$ Rather than a net spin of $20 \mu_{B}$, we predict that the isolated tower structure would have two ferromagnetically ordered inner layers which are antiferromagnetically coupled to the top and bottom layers. The net spin of this tower was found to be zero. These calculations show that in addition to separating the magnetic cores from one another, the nonmagnetic $\mathrm{COOH}$ ligands and $\mathrm{H}_{2} \mathrm{O}$ molecules prevent significant relaxations which would destroy the magnetically interesting $\mathrm{Mn}_{12} \mathrm{O}_{12}$ core.

The electronic structure of this molecule is best described as a half-semiconducting ferrimagnet which is approaching a half-metallic ferrimagnet. The majority spin densities of states shows a small gap of $0.45 \mathrm{eV}$ while the minority spin has a larger gap of $2.08 \mathrm{eV}$. The states near the Fermi level are composed of a mixture of Mn $(3 d)$ electrons and $\mathrm{O}(2 p)$ electrons. As discussed in Ref. 9, these states are very delocalized over the entire molecule and are most responsible for forming the large anisotropy barrier.

For a uniaxial molecule with the axis of symmetry along the $z$ direction, and a magnetic field $\left(B_{z}\right)$ applied along the $z$-direction, the lowest order corrections to the GGA energy are due to interactions of the net moment with the applied field and to the spin-orbit coupling between the electronic and spin degrees of freedom. The energy shift is given by

$$
\Delta=A+\frac{B_{z}}{c}\left\langle S_{z}\right\rangle+\frac{\gamma}{2}\left\langle S_{z}\right\rangle^{2} .
$$

As shown in Ref. 10, the value of $\gamma$ may be calculated within a density functional framework using second-order perturbation theory and, in terms of the Kohn-Sham orbitals, it is given by

$$
\begin{aligned}
\gamma= & \frac{2}{\Delta N^{2}}\left(M_{z z}^{11}+M_{z z}^{22}+M_{x x}^{12}+M_{x x}^{21}-M_{x x}^{11}\right. \\
& \left.-M_{x x}^{22}-M_{z z}^{12}-M_{z z}^{21}\right),
\end{aligned}
$$

where the second-order anisotropy matrices $\left(M_{x y}\right)$ are determined from the following expressions:

$$
\begin{aligned}
& M_{x y}^{\sigma \sigma^{\prime}}=-\sum_{i j} \frac{\left\langle\phi_{i \sigma}\left|V_{x}\right| \phi_{j \sigma^{\prime}}\right\rangle\left\langle\phi_{j \sigma^{\prime}}\left|V_{y}\right| \phi_{i \sigma}\right\rangle}{\epsilon_{i \sigma}-\epsilon_{j \sigma^{\prime}}}, \\
&\left\langle\phi_{i \sigma}\left|V_{x}\right| \phi_{j \sigma^{\prime}}\right\rangle= \frac{1}{2 c^{2}}\left(\left\langle\frac{d \phi_{i \sigma}}{d z}|\Phi| \frac{d \phi_{j \sigma^{\prime}}}{d y}\right\rangle\right. \\
&\left.-\left\langle\frac{d \phi_{i \sigma}}{d y}|\Phi| \frac{d \phi_{j \sigma^{\prime}}}{d z}\right\rangle\right) .
\end{aligned}
$$

In the above expression, $\phi_{i \sigma}$ and $\phi_{j \sigma^{\prime}}$ are, respectively, occupied and unoccupied Kohn-Sham orbitals, $\Phi(\mathbf{r})$ is the Coulomb potential due to the nuclear and electronic degrees of freedom, and $\Delta N$ is the total number of unpaired electrons. As detailed in Ref. 10, the quantities $\left\langle\phi_{i \sigma}\left|V_{x}\right| \phi_{j \sigma^{\prime}}\right\rangle$ represent the exact spatial matrix elements of the spin-orbitcoupling operator $\left[U(\mathbf{r}, \mathbf{p}, \mathbf{S})=-\left(1 / 2 c^{2}\right) \mathbf{S} \cdot \mathbf{p} \times \nabla \Phi(\mathbf{r})\right]$. This expression for the spin-orbit coupling operator is valid for both finite and periodic systems. A positive $\gamma$ corresponds to an easy plane while a negative $\gamma$ corresponds to an easy axis. 
TABLE II. Second order contributions to the magnetomolecular anisotropy energy vs unoccupied-state cut off energy ( $\left.E_{\text {cut }}\right)$ for perturbative calculation. $E_{\text {cut }}$ is measured with respect to the Fermi level. Also included are the number of occupied and unoccupied states used in the sum. The experimental data are from Ref. 7.

\begin{tabular}{lcrc}
\hline \hline \multicolumn{1}{c}{$E_{\text {cut }}(\mathrm{eV})$} & $N_{\text {occ }}$ & $N_{\text {unocc }}$ & Barrier $(\mathrm{K})$ \\
\hline 13.6 & 804 & 730 & 55.7 \\
27.2 & 804 & 1258 & 55.7 \\
13.6 (valence only) & 460 & 730 & 55.7 \\
Experiment & & & 55.6 \\
\hline \hline
\end{tabular}

Assuming $\gamma$ is negative and that the total azimuthal quantum number $M_{S}=\left\langle S_{z}\right\rangle$ the magnetic fields at which spin tunneling occurs are given by integer multiples of the field strength $B_{0}=\gamma c / 2$. Further the second-order anisotropy energy is given by $\gamma \times S^{2} / 2$ with $S$ the total spin of the system. For the $\mathrm{Mn}_{12} \mathrm{O}_{12}(\mathrm{HOOC})_{16}\left(\mathrm{H}_{2} \mathrm{O}\right)_{4}$ molecule we have calculated $\gamma$ using the above formalism. As shown in Table II, our calculated value for the quadratic term gives a barrier of $100(\gamma / 2)=55.7 \mathrm{~K}$. Recently Barra et al. have analyzed electron paramagnetic resonance data and have determined both the quadratic and higher-order contributions to the anisotropy energy. Their value of $\gamma / 2$ (referred to as $\alpha$ in Ref. 6) is found to be $0.556 \mathrm{~K}$. Thus the experimentally determined second order contributions to the anisotropy energies are $55.6 \mathrm{~K}$ in excellent agreement with our calculated value.

In Table II, we show that our calculated anisotropy barrier is well converged with respect to the number of unoccupied states included in the perturbation series. Further exclusion of the core states in the occupied state summation does not affect the value of the anisotropy barrier. We have determined that it is the 160 occupied and 116 unoccupied states that lie within $4.0 \mathrm{eV}$ of the Fermi level that account for $98 \%$ of the anisotropy barrier. This is not unexpected since the pertubative expression shows that pairs of unoccupied and occupied orbitals with similar energies lead to small energy denominators and a potentially large contribution to the perturbative summation. However, contrary to what is expected from considering only energy denominators, our calculations show that the matrix elements between occupied majority spin electrons and unoccupied minority spin electrons that account for $65 \%$ of the second-order anisotropy barrier. The matrix elements between occupied minority-spin electrons and unoccupied majority-spin electrons account for $21 \%$ of the barrier. The smallest energy denominators in these sums are $0.89 \mathrm{eV}$ and $1.63 \mathrm{eV}$, respectively, which is significantly larger than the smallest energy gap (majority-majority) of $0.45 \mathrm{eV}$ in the problem. Even with this small gap, interactions between the majority occupied and majority unoccupied electrons contribute only $13 \%$ to the anisotropy barrier.
The interactions between the minority unoccupied and minority occupied electrons, for which the smallest energy denominator is $2.08 \mathrm{eV}$, contribute only $1 \%$ to the anisotropy barrier. This analysis shows that while energy denominators are important, it is a complicated gradient-weighted spatial overlap integral between occupied and unoccupied states of different spins that are most important for forming the anisotropy barrier in the $\mathrm{Mn}$-acetate molecule. To increase the size of the barrier one would want to concentrate on further enhancing the spatial overlap between the majority occupied and minority unoccupied electrons or by decreasing the energy denominators between these states.

It is indeed the second-order anisotropy energy in uniaxial systems that is the most interesting figure of merit from a technological viewpoint. For this system the Arrhenius data from experiments predicts a total barrier for 60-62 $\mathrm{K}$ which is only $10 \%$ larger than the second-order barrier determined here. For uniaxial systems the higher-order contributions to the barrier are due to several fourth-order effects which include quantization-axis dependent self-consistent effects, additional effects due entirely to the spin-orbit operator, and effects due to higher-order coupling between the spin-orbit operator and scalar relativistic terms. We have determined by direct diagonalization of the Hamiltonian with the exact spin-orbit operator that the fourth order effects due entirely to spin-orbit coupling are negligible.

This work was supported in part by ONR NOOO1498WX20709 (M.R.P.) and DOE DE-FG0296ER45579 (S.N.K.).

${ }^{1}$ T. Lis, Acta Crystallogr., Sect. B: Struct. Crystallogr. Cryst. Chem. 36, 2042 (1980).

${ }^{2}$ J. R. Friedman, M. P. Sarachik, J. Tejada, and R. Ziolo, Phys. Rev. Lett. 76, 3830 (1996)

${ }^{3}$ L. Thomas, F. Lionti, R. Ballou, D. Gatteschi, R. Sessoli, and B. Barbara, Nature (London) 383, 145 (1996)

${ }^{4}$ A. Caneschi, D. Gatteschi, and R. Sessoli, J. Am. Chem. Soc. 113, 5873 (1991)

${ }^{5}$ R. Sessoli, D. Gatteschi, A. Caneshi, and M. A. Novak, Nature (London) 365, 141 (1993).

${ }^{6}$ A. L. Barra, D. Gatteschi, and R. Sessoli, Phys. Rev. B 56, 8192 (1997).

${ }^{7}$ A. Fort, A. Rettori, J. Villain, D. Gatteschi, and R. Sessoli, Phys. Rev. Lett. 80, 612 (1998).

${ }^{8}$ M. R. Pederson and S. N. Khanna, Phys. Rev. B 59, R691 (1999).

${ }^{9}$ M. R. Pederson and S. N. Khanna, Chem. Phys. Lett. 303, 373 (1999).

${ }^{10}$ M. R. Pederson and S. N. Khanna, Phys. Rev. B 60, 9566 (1999).

${ }^{11}$ M. I. Katsnelson, V. V. Dobrovitski, and B. N. Harmon, Phys. Rev. B 59, 6919 (1999).

${ }^{12}$ Z. Zeng, D. Guenzburger, and D. E. Ellis, Phys. Rev. B 59, 6927 (1999).

${ }^{13}$ J. P. Perdew, K. Burke, and M. Ernzerhof, Phys. Rev. Lett. 77, 3865 (1996).

${ }^{14}$ M. R. Pederson and K. A. Jackson, Phys. Rev. B 41, 7453 (1990); K. A. Jackson and M. R. Pederson, ibid. 42, 3276 (1990); D. V. Porezag, Ph.D. thesis, Chemnitz Technical Institute, 1997.

${ }^{15}$ P. J. Ziemann and A. W. Castleman, Jr., Phys. Rev. B 46, 13480 (1992). 